\title{
A Rare Case of Multiple Cranial Nerve Palsies as the First Presentation of Hepatocellular Carcinoma: A Case Report and Review of Literature
}

\author{
Authors: \\ Vedpal Singh Punia, Naman Bansal, *Apoorva Shetty, Nalini Kurri, \\ Neelabh Pratap \\ Department of Medicine, School of Medical Sciences and Research, Sharda University, \\ Greater Noida, India \\ *Correspondence to appzshetty@gmail.com \\ Disclosure: The authors have declared no conflicts of interest. \\ Received: \\ 27.09.21 \\ Accepted: \\ 17.01.22 \\ Keywords: \\ Hepatocellular carcinoma (HCC), multiple cranial nerve palsy, palliative care, portal \\ vein thrombosis, triple-phase CT. \\ Citation: \\ EMJ Neurol. 2022; DOI/10.33590/emjneurol/21-00212.
}

\begin{abstract}
Hepatocellular carcinoma (HCC) is the most common type of primary liver cancer. It mainly occurs in patients with chronic liver diseases such as cirrhosis caused by hepatitis B or C infections, as well as chronic alcoholics. HCC is one of the highly malignant neoplasms. Extrahepatic metastases are seen in $64 \%$ of patients with HCC, but metastases to the brain and skull are extremely rare. Here, the case of a 45-year-old male who presented with left III, IV, VI, IX, X, and XII cranial nerve palsies is reported. These were revealed to be caused by a distant metastasis from an unnoticed HCC with a background of previously undiagnosed chronic alcoholic liver disease. Physicians should be aware of the metastatic potential of HCC, in addition to its uncommon presentations, while encountering patients with an underlying liver disease. This case report demonstrates that metastatic HCC can lead to a rare, but possible, new onset of cranial neuropathy.
\end{abstract}

\section{INTRODUCTION}

The highest incidence rates of hepatocellular carcinoma (HCC) are found in Asia and subSaharan Africa. Overall, liver cancer accounts for $7 \%$ of all cases of cancer (approximately 850,000 new cases each year), while HCC, which frequently metastasises to the lungs, regional lymph nodes, and the skeletal system, represents $90 \%$ of primary liver cancers. Cirrhosis from any aetiology is the strongest risk factor for HCC, and most cases of HCC are C) or alcoholic cirrhosis. HCC in a young patient with no previous evidence of cirrhosis or fibrosis is a relatively rare condition. In an analysis of 770,000 cases of HCC occurring worldwide, over $50 \%$ of cases were attributed to chronic hepatitis B, and $20 \%$ of cases were attributed to chronic hepatitis $C$ infection. ${ }^{2}$ Alcohol-related liver disease is the most prevalent type of chronic liver disease (CLD) worldwide, accounting for $30.0 \%$ of HCC cases, whilst alcoholic liver disease is responsible for $5.4 \%$ of HCC-related deaths. ${ }^{3}$ 
An excessive alcohol intake may result in fatty liver, acute or chronic hepatitis, and/or cirrhosis, and eventually lead to HCC. Extrahepatic metastases are seen in $64.0 \%$ of patients, while brain metastases from HCC are extremely rare, with a reported frequency of $0.2-2.2 \%{ }^{4-7}$ The interval between the diagnosis of primary cancer and the detection of a brain metastasis ranged from 2 months to 54 months; the mean survival period was only 3 months after the diagnosis of brain metastasis. The attributed cause of death in patients with HCC with a metastatic brain is, therefore, neurological failure rather than hepatic failure.

\section{CASE REPORT}

This case report describes a 45-year-old male patient from Uttar Pradesh, India, who presented to the authors' medical outpatient department with a history of fever that had a usual duration of 1 day. Upon further evaluation, the patient also reported a history of headaches, tongue deviation towards the left side, hoarseness of voice, difficulty swallowing, and drooping of the left eyelid over the preceding 2 months. The patient also described a loss of appetite for the last 2 months, which caused them to lose approximately $20 \mathrm{~kg}$ of weight. He denied having any evidence of a pre-existing liver disease; however, he used to drink alcohol occasionally. He was a non-smoker and denied any usage of intravenous recreational drugs.
The patient gave no history of chronic medical illness or any significant family history. He had not undergone any surgical procedures in the past or received any blood transfusions.

On physical examination, the patient appeared moderately built, poorly nourished, fully conscious with a Glasgow coma scale (GCS) score of $15 / 15$, and oriented to time, place, and person, with no cognitive impairment. He had presented with pyrexia on admission, with a body temperature of $101^{\circ} \mathrm{F}$, but displayed no pallor, icterus, clubbing, cyanosis, or oedema. On systemic examination, an abdominal examination revealed distension of the abdomen with no stigmata of CLD or any peripheral signs of hepatic failure. A respiratory examination revealed bibasilar crepitations. The cardiovascular examination was unremarkable.

A neurological examination revealed ptosis of the left eye, restricted left eyeball movement, left side soft palatal movement, dysphagia, hoarseness, and deviation of the tongue to the left side with left vocal cord palsy, which suggested that the patient had left III, IV, VI, IX, X, and XII cranial nerve palsies. Other cranial nerve examinations, sensory functions, motor functions, and cerebellar functions were all normal. The laboratory studies of the patient's blood that were undertaken on admission are seen in Tables 1 and 2. A serological examination for viral hepatitis, including hepatitis $B$ and $C$, was negative, and the results from the iron panel test, renal function test, and electrolyte test were all normal.

Table 1: Abnormal liver function test with a reference range.

\begin{tabular}{|l|l|l|}
\hline Test name & Result value & Reference range \\
\hline Total bilirubin & $2.0 \mathrm{mg} / \mathrm{dL}$ & $0.2-1.3 \mathrm{mg} / \mathrm{dL}$ \\
\hline Direct bilirubin & $1.14 \mathrm{mg} / \mathrm{dL}$ & $0-0 \mathrm{mg} / \mathrm{dL}$ \\
\hline Indirect bilirubin & $1.08 \mathrm{mg} / \mathrm{dL}$ & $0.00-1.00 \mathrm{mg} / \mathrm{dL}$ \\
\hline SGOT & $216 \mathrm{U} / \mathrm{L}$ & $0-40 \mathrm{U} / \mathrm{L}$ \\
\hline SGPT & $98 \mathrm{U} / \mathrm{L}$ & $0-50 \mathrm{U} / \mathrm{L}$ \\
\hline Alkaline phosphate & $629 \mathrm{U} / \mathrm{L}$ & $38-126 \mathrm{U} / \mathrm{L}$ \\
\hline Serum albumin & $2.8 \mathrm{~g} / \mathrm{L}$ & $3.5-5.0 \mathrm{mg} / \mathrm{dL}$ \\
\hline PT/INR & $14.0 \mathrm{sec} / 1.7$ & $9.5-16.5 \mathrm{sec} / 1.0$ \\
\hline
\end{tabular}

INR: international normalised ratio; PT: prothrombin time; SGOT: serum glutamic-oxaloacetic transaminase; SGPT: serum glutamic-pyruvic transaminase. 
Table 2: Test values for a-fetoprotein, erythrocyte sedimentation rate, and D-dimer with a reference range.

\begin{tabular}{|l|l|l|}
\hline Test name & Result value & Reference range \\
\hline a-fetoprotein & $65,000 \mathrm{IU} / \mathrm{mL}$ & $<7 \mathrm{IU} / \mathrm{mL}$ \\
\hline ESR & $35 \mathrm{~mm} / \mathrm{hour}$ & $0-15 \mathrm{~mm} / \mathrm{hour}$ \\
\hline D-dimer & $>4 \mu \mathrm{g} / \mathrm{L}$ & $0.0-0.5 \mu \mathrm{g} / \mathrm{L}$ \\
\hline
\end{tabular}

ESR: erythrocyte sedimentation rate.

A triple-phase abdominal CT scan of the liver was performed (including an arterial phase, a portal venous phase, and a late washout phase). The scan showed hepatomegaly with multiple hypodense irregular lesions, with the largest lesion in right lobe showing enhancement on arterial phase (Figure 1). The imaging and washout on portal venous phase (Figure 2) were suggestive of HCC with metastasis and CLD. The dilated portal vein appeared with an intraluminal filling defect in intra- and extrahepatic segments involving both its branches, showing a post-contrast enhancement that was suggestive of portal vein thrombosis with portal hypertension and moderate ascites.

An MRI scan of the brain (Figure 3) revealed a well-circumscribed, rounded lesion adjacent to the left cerebellopontine angle, abutting the medulla medially and causing mild compression over it. The enhancement that showed on post-contrast imaging was suggestive of a brain metastasis.

A high-resolution CT scan of the chest (Figure 4) revealed a hyperdense, wellcircumscribed lesion with irregular margins in the posterior basal segment of the left lower lobe peripherally, showing mild post-contrast enhancement that was likely to be suggestive of pulmonary metastasis.

\section{DISCUSSION}

HCC is one of the most common causes of cancer-related deaths worldwide. ${ }^{8}$ The presence of brain metastases is associated with significant morbidity and mortality, and considerable research is focused on improving both the survival and quality of life of these patients. Brain metastases are most frequently diagnosed in patients with lung, breast, and melanoma primary tumours. ${ }^{9}$ However, brain metastases from HCC is extremely rare, with a reported frequency of $0.2-2.2 \%$ at autopsy. ${ }^{5-8}$ The prognosis of the patients with a brain metastasis is extremely poor, with a median survival of only 1-2 months. 5,10,11 To date, only a few studies from Asia, as well as some small studies from the USA and Europe, on brain metastases with HCC have been reported. $5,6,10-15$

In the present case, although a histological examination was not performed, the diagnosis was considered to be brain metastases from HCC, based on the following radiological and laboratory findings. Radiological diagnosis is achieved with a high degree of confidence if the lesion is $\geq 2 \mathrm{~cm}$ in diameter and shows the radiological hallmarks of $\mathrm{HCC}$ by one imaging technique. Using contrast-enhanced imaging techniques, the typical hallmark of HCC is the vascular uptake of the nodule in the arterial phase with washout in the portal venous or delayed phases. This radiological pattern captures the hypervascular nature that is characteristic of HCC. In these scenarios, the diagnostic specificity is approximately $95-100 \%$ and a biopsy is not necessary. ${ }^{16}$ a-fetoprotein was taken as an accessory biomarker to aid the diagnosis of HCC. However, a liver biopsy could not be performed in the patient as they did not give consent, which was considered a limitation of the study.

In the patient case outlined here, the findings of the triple-phase abdominal CT scan and the extremely elevated levels of a-fetoprotein $(60,500 \mathrm{lU} / \mathrm{mL})$ suggest a diagnosis of $\mathrm{HCC}$. Patients with HCC are assessed using the Barcelona Clinic Liver Cancer (BCLC) staging system, which incorporates the number and 


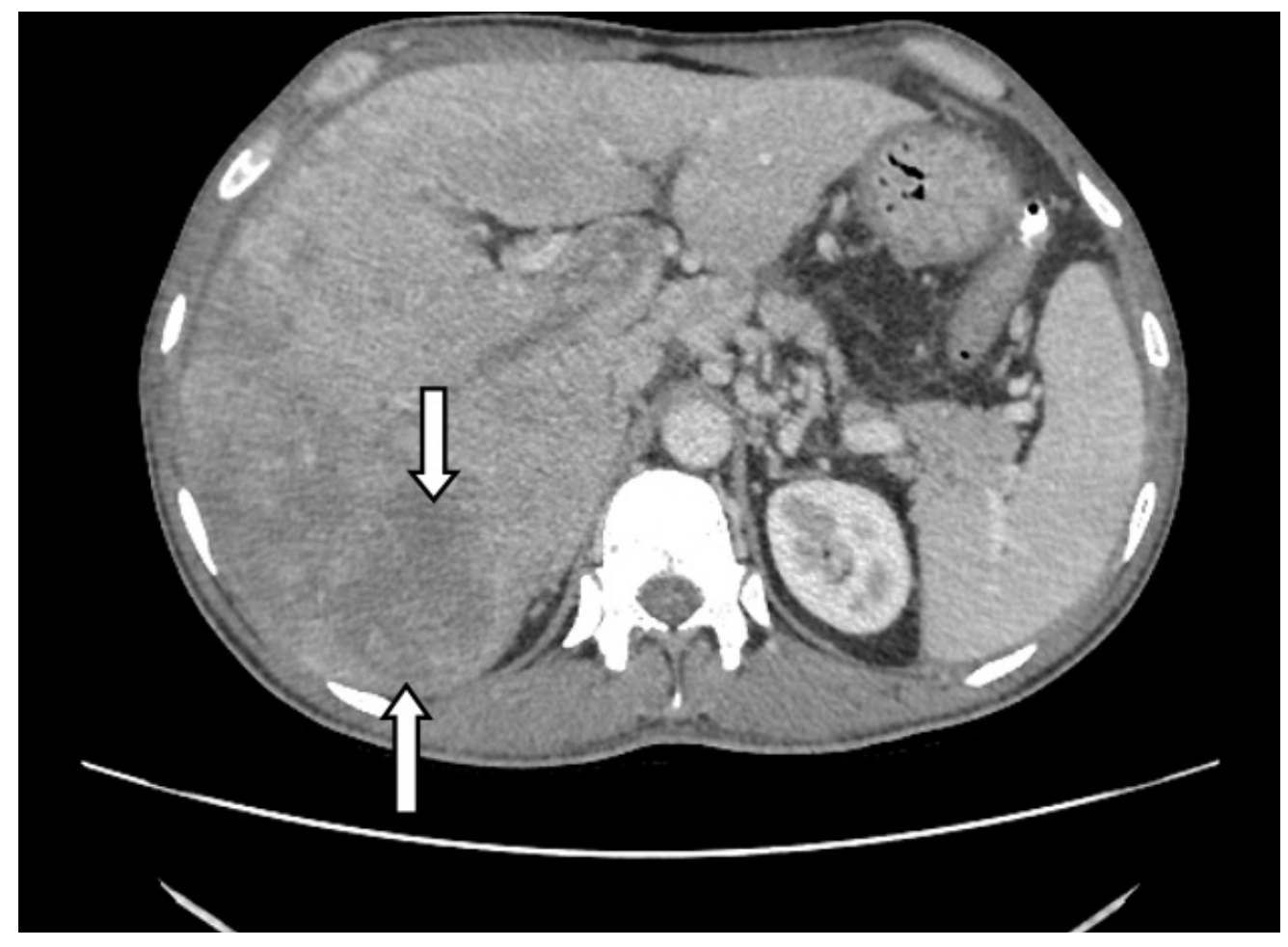

Figure 1: Axial plane of an abdominal CT scan in the arterial phase, showing an irregular iso-hypodense mass lesion (indicated by the white arrows) occupying the right lobe of the liver, with mild contrast enhancement.

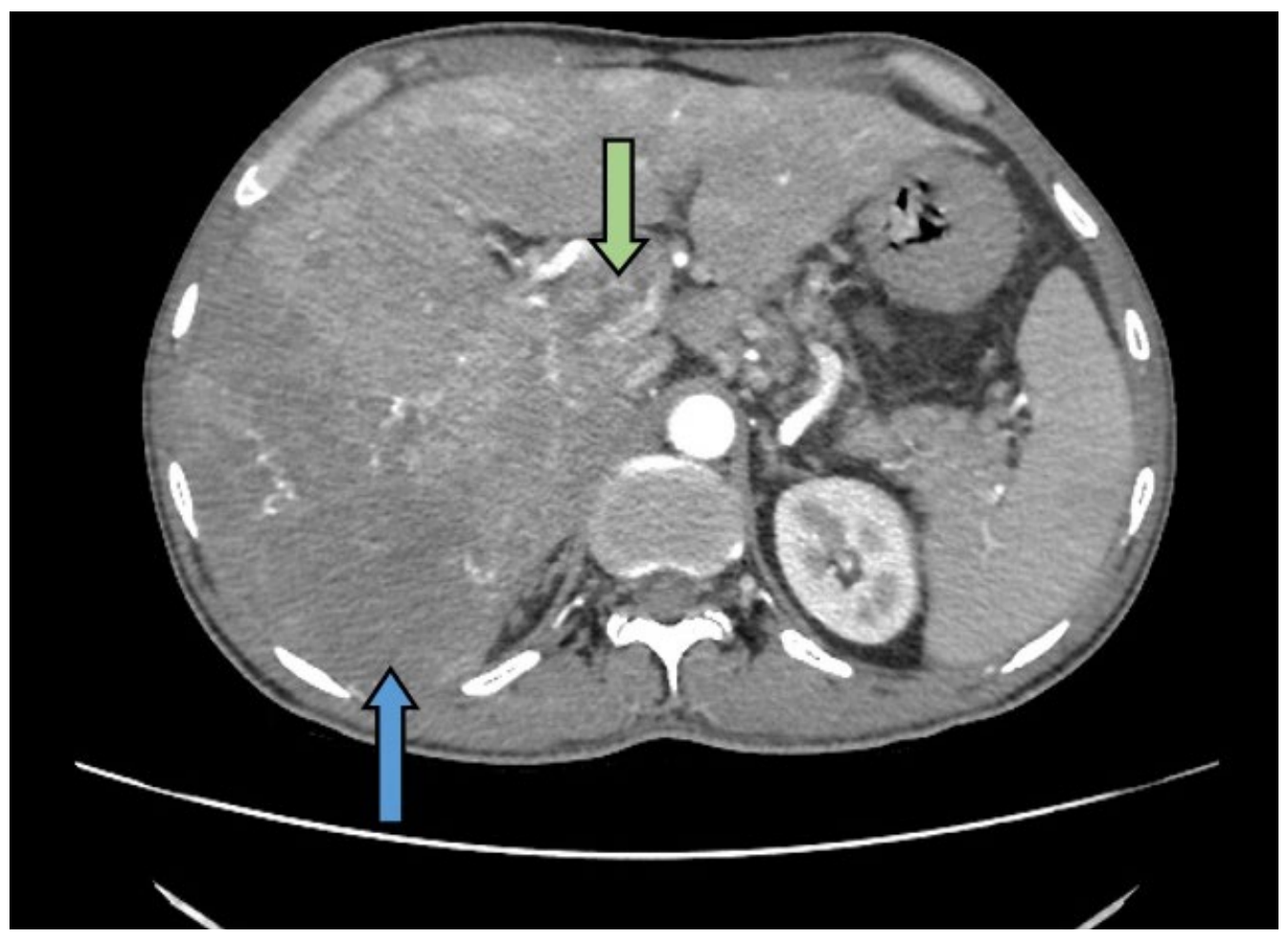

Figure 2: Axial plane of abdominal CT scan in the portal venous phase, showing washout from the mass lesion (blue arrow).

The proximal part of the portal vein is dilated, with an intraluminal filling defect showing post-contrast enhancement (green arrow). 


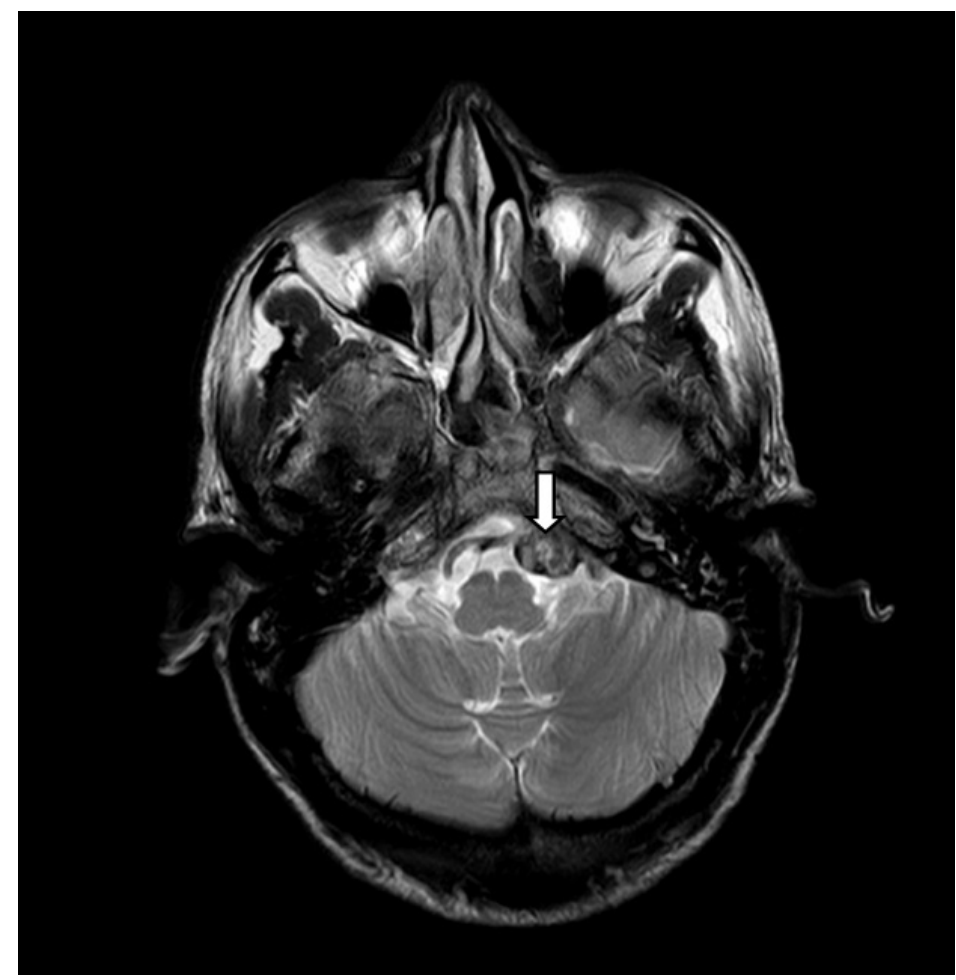

Figure 3: This brain MRI axial section reveals a well-circumscribed rounded lesion (white arrow), adjacent to the left cerebellopontine angle, abutting the medulla medially, causing mild compression on it and showing enhancement on the post-contrast imaging, suggestive of a brain metastasis.

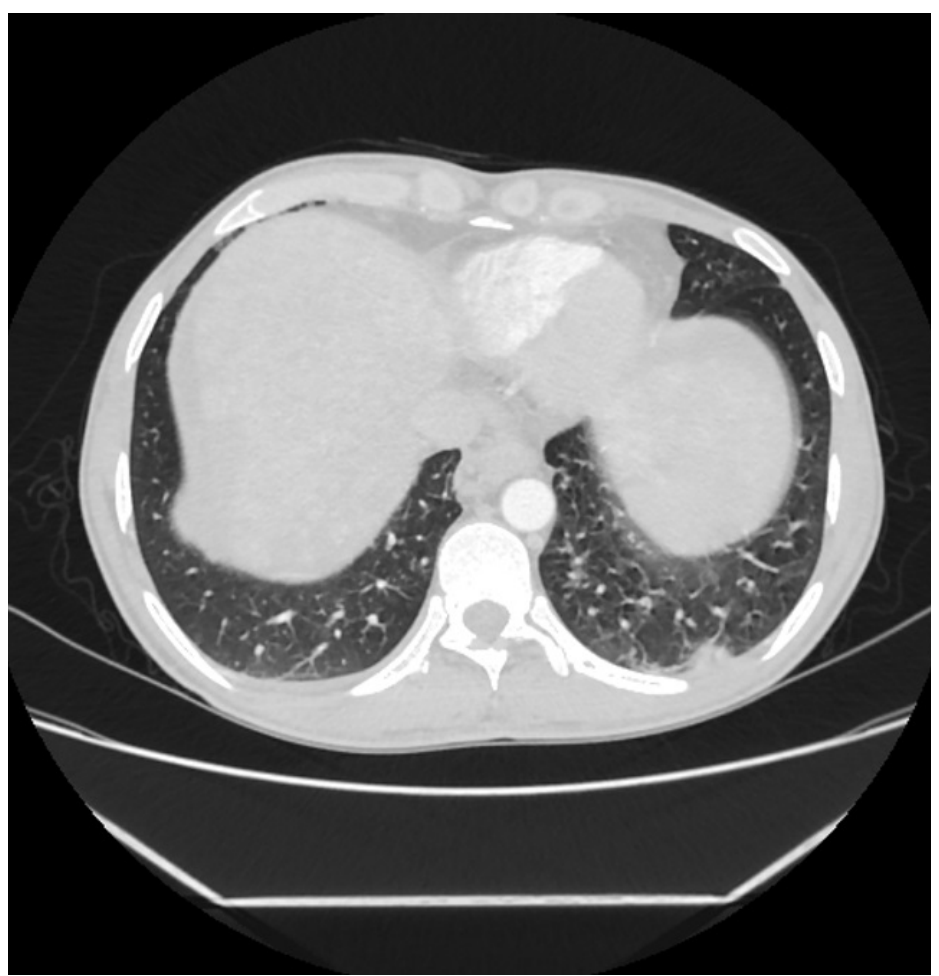

Figure 4: High-resolution chest CT revealing a hyperdense, well-circumscribed lesion with irregular margins in the posterior basal segment of the left lower lobe peripherally, showing mild post-contrast enhancement, likely suggestive of a pulmonary metastasis. 
size of tumours in the liver, performance status, and liver function. A Child-Pugh score was used to assess the liver function, and an Eastern Cooperative Oncology Group (ECOG) score was used to determine performance status. The patient was determined to be at BCLC Stage D, with a Child-Pugh score C, and an ECOG performance status of 3. Patients with BCLC Stage D HCC, which is often referred to as terminal stage, have a median survival of less than 3 months m,9,10 $^{6}$ and can be offered palliative supportive care, as disease-directed treatments are not available for patients with terminal HCC. Palliative care should be offered early in order to meet the complex medical, social, and psychological needs of these patients.

\section{CONCLUSION}

HCC with brain metastases is a life-threatening condition due to the added risk of haemorrhage and the limited treatment options available. Since there is no standard therapeutic strategy available to date, an early diagnosis would certainly improve the chance of curative surgical resection, thus increasing survival in patients with unresectable tumours. The rarity of this presentation with no clinical signs of chronic liver stigmata should alert physicians to be diligent when they are confronted with an abnormal radiological liver finding. There are studies available on brain metastases caused by HCC; however, there are very few studies related to multiple cranial nerve palsies. This case report may, therefore, be of importance due to the rarity of this condition and the need to create awareness in order to prevent deaths from neurological causes in patients with HCC who have metastatic brain tumours.

\section{References}

1. Kashima M et al. Radiofrequency ablation for the treatment of bone metastases from hepatocellular carcinoma. AJR Am J Roentgenol. 2010;194:536-41.

2. Maucort-Boulch D et al. Fraction and incidence of liver cancer attributable to hepatitis B and C viruses worldwide. Int J Cancer. 2018;142(12):2471-7.

3. Paik JM et al. Nonalcoholic fatty liver disease and alcoholic liver disease are major drivers of liver mortality in the United States. Hepatol Commun. 2020;4(6):890-903

4. Kim M et al. Nervous system involvement by metastatic hepatocellular carcinoma. $J$ Neurooncol. 1998;36(1):85-90.

5. Choi HJ et al. Brain metastases from hepatocellular carcinoma: prognostic factors and outcome: brain metastasis from HCC. J Neurooncol. 2009;91(3):307-13

6. Seinfeld $\mathrm{J}$ et al. Brain metastases from hepatocellular carcinoma in US patients. J Neurooncol. 2006;76(1):93-8.

7. Murakami $\mathrm{K}$ et al. Intracranial metastases of hepatocellular carcinoma: CT and MRI. Neuroradiology. 1996;38(Suppl 1):S315.

8. Jemal A et al. Global cancer statistics. CA Cancer J Clin. 2011;61(2):69-90.

9. Sperduto PW et al. Diagnosis-specific prognostic factors, indexes, and treatment outcomes for patients with newly diagnosed brain metastases: a multi-institutional analysis of 4,259 patients. Int J Radiat Oncol Biol Phys. 2010;77(3):655-61.

10. Chang $L$ et al. Intracranial metastasis of hepatocellular carcinoma: review of 45 cases. Surg Neurol. 2004;62(2):172-7.

11. Jung $\mathrm{HH}$ et al. Little response of cerebral metastasis from hepatocellular carcinoma to any treatments. J Korean Neurosurg Soc.
2010;47(5):325-31

12. França AV et al. Brain metastasis of hepatocellular carcinoma detected after liver transplantation. Arq Gastroenterol. 2004;41(3):199-201.

13. Tunc $B$ et al. Brain metastasis of hepatocellular carcinoma: a case report and review of the literature. World J Gastroenterol. 2004;10(11):1688-9.

14. Del Ben M et al. Intracranial hemorrage due to brain metastases in an Italian HCV patient with hepatocellular carcinoma. J Exp Clin Cancer Res. 2003;22(4):641-4.

15. Frati A et al. Brain metastasis from hepatocellular carcinoma associated with hepatitis B virus. J Exp Clin Cancer Res. 2002;21(3):321-7.

16. Yu NC et al. CT and MRI improve detection of hepatocellular carcinoma, compared with ultrasound alone, in patients with cirrhosis. Clin Gastroenterol Hepatol. 2011;9(2):161-7. 